\title{
When is needle examination of thenar muscle necessary in the evaluation of mild and moderate carpal tunnel syndrome?
}

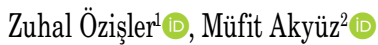 \\ ${ }^{1}$ Department of Physical Medicine and Rehabilitation, Ankara City Hospital, Ankara, Turkey \\ ${ }^{2}$ Department of Physical Medicine and Rehabilitation, Karabuk University, Faculty of Medicine, Karabuk, Turkey
}

Received: March 11, 2021 Accepted: September 08, 2021 Published online: December 01, 2021

\begin{abstract}
Objectives: This study aims to evaluate the predictors of standard nerve conduction study (NCS) parameters in determining the presence of axonal loss by means of spontaneous activity in patients with mild and moderate carpal tunnel syndrome (CTS).

Patients and methods: Between May 2015 and April 2018, a total of 118 patients (11 males, 107 females; mean age: $52.3 \pm 10.6$ years; range, 27 to 79 years) who underwent electrophysiological studies and were diagnosed with CTS were included. Demographic data of the patients including age, sex, and symptom duration were recorded. Electrodiagnostic studies were performed in all patients. All the needle electromyography (EMG) findings were recorded, but only the presence or absence of spontaneous EMG activities was used as the indicator of axonal injury.

Results: In 37 (31.4\%) of the patients, spontaneous activity was detected at the thenar muscle needle EMG. No spontaneous activity was observed in any of 43 (36.4\%) patients with normal distal motor latency (DML). There were significant differences in DMLs, compound muscle action potential (CMAP) amplitudes, sensory nerve action potentials amplitudes, and sensory nerve conduction velocities between the groups with and without spontaneous activity $(\mathrm{p}<0.05)$. The multiple logistic regression analysis revealed that DML was a significant independent risk variable in determining presence of spontaneous activity. The most optimal cut-off value for median DML was calculated as $4.9 \mathrm{~ms}$. If the median DML was $>4.9 \mathrm{~ms}$, the relative risk of finding spontaneous activity on thenar muscle needle EMG was 13.5 ( $95 \%$ CI: 3.6-51.2).
\end{abstract}

Conclusion: Distal motor latency is the main parameter for predicting the presence of spontaneous activity in mild and moderate CTS patients with normal CMAP. Performing needle EMG of the thenar muscle in CTS patients with a DML of $>4.9$ ms may be beneficial to detect axonal degeneration in early stages.

Keywords: Carpal tunnel syndrome, needle electromyography, spontaneous activity, thenar muscle.

Carpal tunnel syndrome (CTS) is one of the most common entrapment neuropathies and occurs as a result of chronic compression of the median nerve under the transverse carpal ligament at the wrist. $^{[1-3]}$ It affects approximately 3 to $9.8 \%$ of the general population, although its incidence varies depending on age, sex, and work-related conditions. ${ }^{[4-7]}$ Carpal tunnel syndrome is one of the most frequently studied preliminary diagnoses in electromyography (EMG) laboratories. ${ }^{[8]}$

The diagnosis of CTS is made based on typical symptoms, signs, and provocative tests and electrophysiological study of the median nerve. Electrodiagnostic test is particularly useful to confirm median neuropathy at the wrist, particularly in atypical cases and to evaluate the

Corresponding author: Zuhal Özişler, MD. Ankara Şehir Hastanesi Fizik Tedavi ve Rehabilitasyon Kliniği, 06800 Çankaya, Ankara, Türkiye. 
severity of CTS. ${ }^{[9,10]}$ Carpal tunnel syndrome is primarily a focal demyelinating condition, and nerve conduction studies (NCSs) are usually sufficient for documenting the slowing of conduction at the carpal tunnel. ${ }^{[3,8,11]}$ Therefore, the guidelines of the American Association of Neuromuscular and Electrodiagnostic Medicine (AANEM) do not always consider needle evaluation essential for electrophysiological evaluation of CTS. ${ }^{[12]}$ However, needle evaluation may be required for excluding the differential diagnosis. On the other hand, the determination of the severity of CTS is of utmost importance, as it may influence the clinical treatment approach. Conservative treatment methods can be used in CTS patients without significant denervation. However, in more severe cases with axonal injury, delayed surgical treatment increases the risk of irreversible progressive median nerve injury. ${ }^{[13-15]}$

The NCS can establish the diagnosis, but cannot always be sufficient to identify axonal degeneration. Although the relationship between low-compound muscle action potential (CMAP) amplitude and axonal loss is known, low CMAP amplitude of median nerve can be related to conduction block at the carpal tunnel. On the other hand, normal CMAP amplitude does not always exclude the presence of axonal injury. Although the denervation potential in the thenar muscles is the best evidence to identify the presence of axonal degeneration, needle EMG of thenar muscles is uncomfortable for the patient. It is important to decide performing needle EMG of thenar muscle in electrophysiological evaluation of CTS. However, there are different opinions about the necessity and time of the of the thenar muscle needle examination. ${ }^{[16-18]}$

In particular, in CTS patients with normal or moderately low CMAP amplitude, it is useful to recognize predictive parameters of NCS to perform thenar muscles needle EMG. Such a recognition would be helpful for the practitioner to early detect axonal degeneration and to avoid the patient from an unnecessary painful procedure. In the present study, we aimed to evaluate the predictors of standard NCS parameters in determining the presence of axonal loss by means of spontaneous activity in patients with mild and moderate CTS.

\section{PATIENTS AND METHODS}

This prospective observational study was conducted at Ankara Physical Medicine and Rehabilitation Training and Research Hospital, Department of Physical Medicine and Rehabilitation between May
2015 and April 2018. Patients who were referred to the our EMG laboratory for electrophysiological testing for CTS and who met the CTS diagnostic criteria were included in the study. The electrophysiological criteria used for diagnosis of CTS were the presence of slowed sensory median conduction velocities at wrist with/without prolonged median distal motor latency (DML). More severe patients with CMAP amplitude $<4 \mathrm{mV}$ and/or those with unobtainable both median sensory potentials (palm-wrist and second digit-wrist) were excluded from the study. Patients with accompanying ulnar neuropathy, radiculopathy, and polyneuropathy as confirmed by electrophysiological studies were also excluded. Finally, a total of 118 patients with CTS (11 males, 107 females; mean age: $52.3 \pm 10.6$ years; range, 27 to 79 years) were included. A written informed consent was obtained from each patient. The study protocol was approved by the Ankara Physical Medicine and Rehabilitation Training and Research Hospital Ethics Committee (2015/281). The study was conducted in accordance with the principles of the Declaration of Helsinki.

Demographic data of the patients including age, sex, and symptom duration were recorded. Electrodiagnostic studies were performed using a Dantec ${ }^{\mathrm{TM}}$ Keypoint $^{\circledR}$ EMG device (Medtronic Functional Diagnostics A/S, Denmark) by the same researcher. Surface electrodes were used for NCSs. For motor conduction study of median nerve, recording electrode was placed to the abductor pollicis brevis (APB) and stimulations were given at the wrist and the cubital fossa. The DML, CMAP amplitude, and forearm motor nerve conduction velocity (FMCV) were calculated. For motor conduction study of ulnar nerve, a recording electrode was placed to the abductor digiti minimi (ADM) and stimulations were given at the wrist, below and above the elbow. The ratio of median and ulnar CMAP (median/ulnar CMAP) were calculated. Median and ulnar sensory conduction studies were performed by orthodromic stimulation. Recording electrodes were placed on the wrist. Median nerve was stimulated at the palm and the second digit. Ulnar nerve was stimulated at the fifth digit. Sensory nerve action potential (SNAP) amplitudes and sensory nerve conduction velocities (distance/peak latency of SNAP) were calculated.

Needle EMG of the APB muscles was performed in all of the patients. All needle examination was performed with disposable concentric needles by using a standard-needle EMG technique. All the needle 
EMG findings were recorded, but only the presence or absence of spontaneous EMG activities was used as the indicator of axonal injury.

\section{Statistical analysis}

Statistical analysis was performed using the IBM SPSS version 25.0 software (IBM Corp., Armonk, NY, USA). The Shapiro-Wilk test was used to analyze the distributions of continuous variables. Continuous variables were expressed in mean \pm standard deviation (SD) and median and interquartile range (IQR) $\left(25^{\text {th }}-75^{\text {th }}\right.$ percentiles), while categorical variables were expressed in number and frequency. The mean differences between the groups were compared using the Student t-test, otherwise the Mann-Whitney $U$ test was used for the comparison of non-normally distributed data. The Pearson chi-square test was used to analyze categorical data. In all $2 \times 2$ contingency tables to compare categorical variables, the continuity corrected chi-square test was used, when one or more of the cells had an expected frequency of 5-25; otherwise, the Fisher's exact test was used when one or more of the cells had an expected frequency of 5 or less. The optimal thresholds for electrophysiological measures to discriminate patients with and without spontaneous activity on thenar muscle needle EMG were evaluated using the receiver operating characteristic (ROC) analysis via Youden's index (J). Any threshold which was given the maximum sum of sensitivity and specificity for the significant test was considered as the optimal cut-off point according to the $J$ statistic (i.e., $J=$ Sensitivity + Specificity - 1). Sensitivity, specificity, positive predictive value (PPV), and negative predictive value (NPV) for each significant electrophysiological measurement were also calculated. The most significant independent predictors to distinguish patients with and without spontaneous activity were investigated using the multiple logistic regression analysis. Any variable with a $\mathrm{p}$ value of $<0.10$ was accepted as a candidate for the multivariable model. Odds ratios (OR), 95\% confidence intervals (CI), and Wald statistics for each independent variable were also calculated. A $p$ value of $<0.05$ was considered statistically significant.

\section{RESULTS}

Spontaneous activity was detected in 37 (31.4\%) of the patients in the thenar muscle needle EMG. The mean age, mean symptom duration, and sex distribution of the groups with and without spontaneous activity were similar $(\mathrm{p}>0.05)$. Significant differences were found in DMLs, CMAP amplitudes,
palm-to-wrist-SNAP amplitudes (pwSNAP), palm-towrist-SNCVs (pwSNCV), median/ulnar CMAP ratio between CTS patients with and without spontaneous activity $(\mathrm{p}<0.05)$. We could not obtain the second digitSNAPs (2dSNAP) in all of the patients and, therefore, for the analysis of this parameter, the chi square test was used. Demographic and electrophysiological parameters with and without spontaneous activity of the patients are summarized in Table 1.

The DMLs were within normal limits in 43 (36.4\%) of the patients and prolonged in $75(63.6 \%)$ of the patients. No spontaneous activity was observed in any of patients with normal DML. Therefore, subgroup analysis was performed in 75 patients with prolonged DML to identify the spontaneous activity predictors according to the NCSs. Totally, 37 (49.3\%) of 75 CTS patients with prolonged DMLs had spontaneous activity. In CTS patients with prolonged DMLs, there were significant differences in DMLs, CMAP amplitudes, pwSNAP amplitudes, and pwSNCVs between the groups with and without spontaneous activity $(\mathrm{p}<0.05)$; however, there were no significant difference in the median/ulnar CMAP ratio and FMCVs $(p>0.05)$. The electrophysiological parameters with and without spontaneous activity in CTS patients with prolonged DMLs are summarized in Table 2. Seventeen $(73.9 \%)$ of 23 CTS patients with unobtainable 2dSNAPs amplitudes had spontaneous activity. The CTS patients whose the 2dSNAPs amplitude was unobtainable were 12 times (95\% CI: 2.9-50.5) more likely to present spontaneous activity on thenar muscle needle EMG than the cases whose 2dSNAPs amplitude was normal.

According to the ROC analysis, the area under the ROC curve of DML, pwSNAP amplitude and pwSNCV measurements were found to be statistically significant in distinguishing patients with and without spontaneous activity $(\mathrm{p}<0.005)$ (Figure 1$)$. We found the most optimal cut-off value for DML to be $4.9 \mathrm{~ms}$ in discriminating the presence of spontaneous activity. The sensitivity of DML at this point was $78.4 \%$ and the specificity was $84.2 \%$. The PPV and NPV of the DML cut-off were $82.9 \%$ and $80.0 \%$, respectively. We found the most optimal cut-off value for pwSNAP amplitude to be $<22.5 \mu \mathrm{V}$ and pwSNCV to be $<25.6 \mathrm{~m} / \mathrm{s}$ in discriminating the presence of spontaneous activity (Table 3).

The multiple logistic regression analysis was performed to identify the most optimal predictors to distinguish patients with and without spontaneous activity on needle EMG. Accordingly, the DML was a 


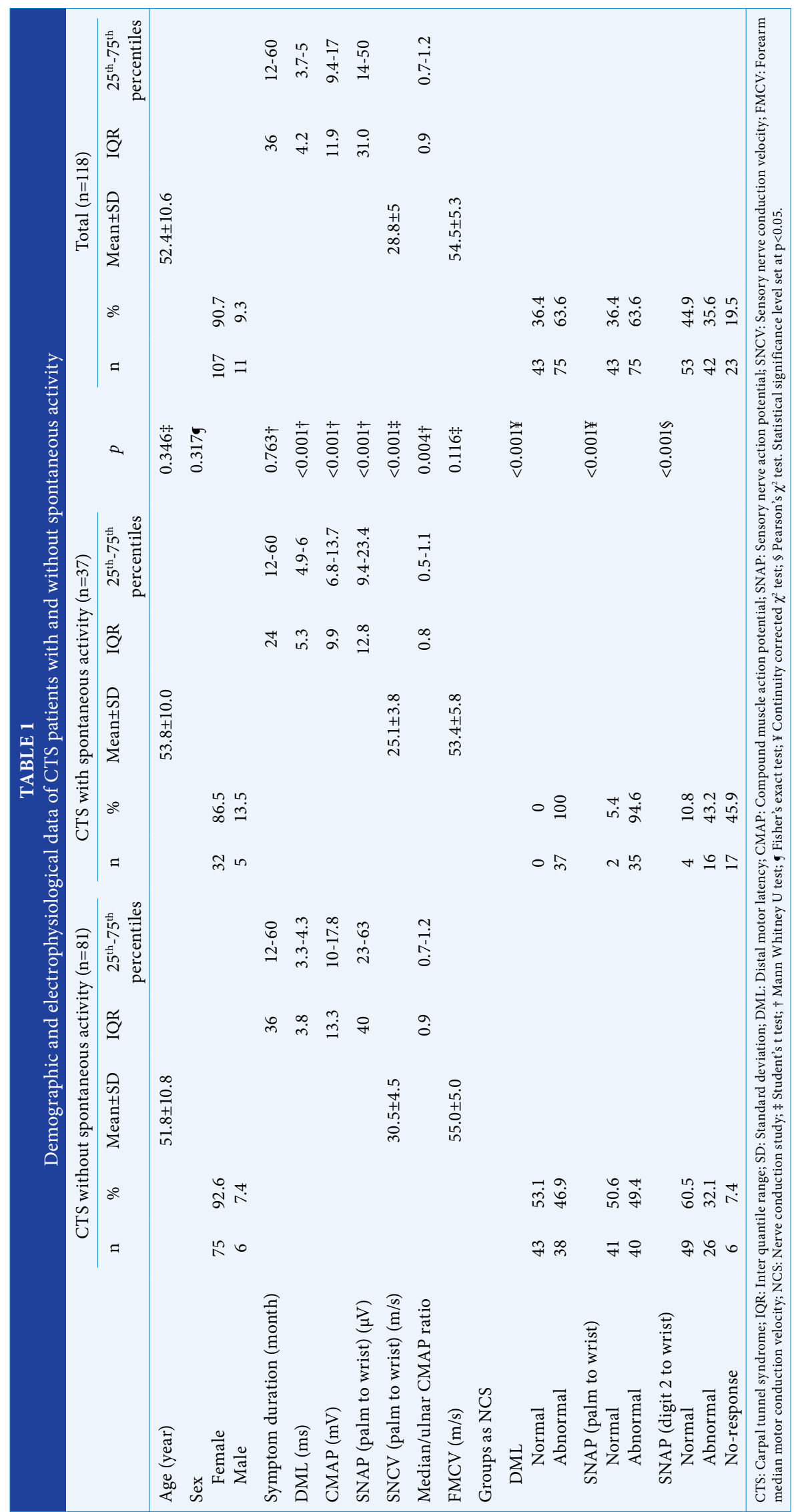




\begin{tabular}{|c|c|c|c|c|c|c|c|c|}
\hline \multicolumn{9}{|c|}{ Electrophysiological data of groups with or without sp } \\
\hline 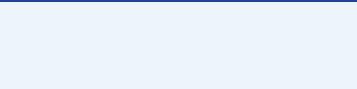 & \multicolumn{3}{|c|}{$\begin{array}{l}\text { CTS without spontaneous } \\
\text { activity }(\mathrm{n}=38)\end{array}$} & \multicolumn{3}{|c|}{$\begin{array}{l}\text { CTS with spontaneous } \\
\text { activity }(n=37)\end{array}$} & \multirow[b]{2}{*}{$\begin{array}{l}\text { Mean differences } \\
\quad(95 \% \mathrm{CI})\end{array}$} & \multirow[b]{2}{*}{$p$} \\
\hline & Mean \pm SD & IQR & $\begin{array}{c}25^{\text {th }}-75^{\text {th }} \\
\text { percentiles }\end{array}$ & Mean \pm SD & IQR & $\begin{array}{c}25^{\text {th }}-75^{\text {th }} \\
\text { percentiles }\end{array}$ & & \\
\hline DML (ms) & & 4.3 & $4.2-4.7$ & & 5.3 & $4.9-6$ & NA & $<0.001 \dagger$ \\
\hline CMAP (mV) & & 12 & $9.4-17.8$ & & 9.9 & $6.8-13.7$ & NA & $0.015 \dagger$ \\
\hline SNCV (palm to wrist) (m/s) & $28.1 \pm 4.9$ & & & $25.1 \pm 3.8$ & & & $-3.04(-5.05--1.03)$ & $0.004 \ddagger$ \\
\hline SNAP (palm to wrist) $(\mu \mathrm{V})$ & & 27 & $14-43.5$ & & 12.8 & $9.4-23.4$ & NA & $<0.001 \dagger$ \\
\hline Median/ulnar CMAP ratio & & 0.9 & $0.6-1.1$ & & 0.8 & $0.5-1.1$ & NA & $0.198 \dagger$ \\
\hline $\operatorname{FMCV}(\mathrm{m} / \mathrm{s})$ & $53.3 \pm 4.6$ & & & $53.4 \pm 5.8$ & & & $0.06(-2.3-2.5)$ & $0.960 \ddagger$ \\
\hline
\end{tabular}

\begin{tabular}{|c|c|c|c|c|c|c|c|c|}
\hline \multicolumn{9}{|c|}{$\begin{array}{c}\text { TABLE } 3 \\
\text { The diagnostic performance indicators of electrophysiological data in distinguishing patients with and without spontaneous } \\
\text { activity in CTS }\end{array}$} \\
\hline & \multicolumn{2}{|c|}{ Sensitivity } & \multicolumn{2}{|c|}{ Specificity } & \multicolumn{2}{|c|}{ PPV } & \multicolumn{2}{|c|}{ NPV } \\
\hline & $\%$ & $95 \% \mathrm{CI}$ & $\%$ & $95 \% \mathrm{CI}$ & $\%$ & $95 \% \mathrm{CI}$ & $\%$ & $95 \% \mathrm{CI}$ \\
\hline $\mathrm{DML}>4.9 \mathrm{~ms}$ & 78.4 & $65.1-91.6$ & 84.2 & $72.6-95.8$ & 82.9 & $70.4-95.3$ & 80.0 & $67.6-92.4$ \\
\hline SNAP (palm to wrist) $<22.5 \mu \mathrm{V}$ & 75.7 & $61.8-89.5$ & 63.2 & $47.8-78.5$ & 66.7 & $52.4-80.9$ & 72.7 & $57.5-87.9$ \\
\hline SNCV (palm to wrist) $<25.6 \mathrm{~m} / \mathrm{s}$ & 56.8 & $40.8-72.7$ & 73.7 & $59.7-87.7$ & 67.7 & $51.3-84.2$ & 63.6 & $49.4-77.8$ \\
\hline
\end{tabular}

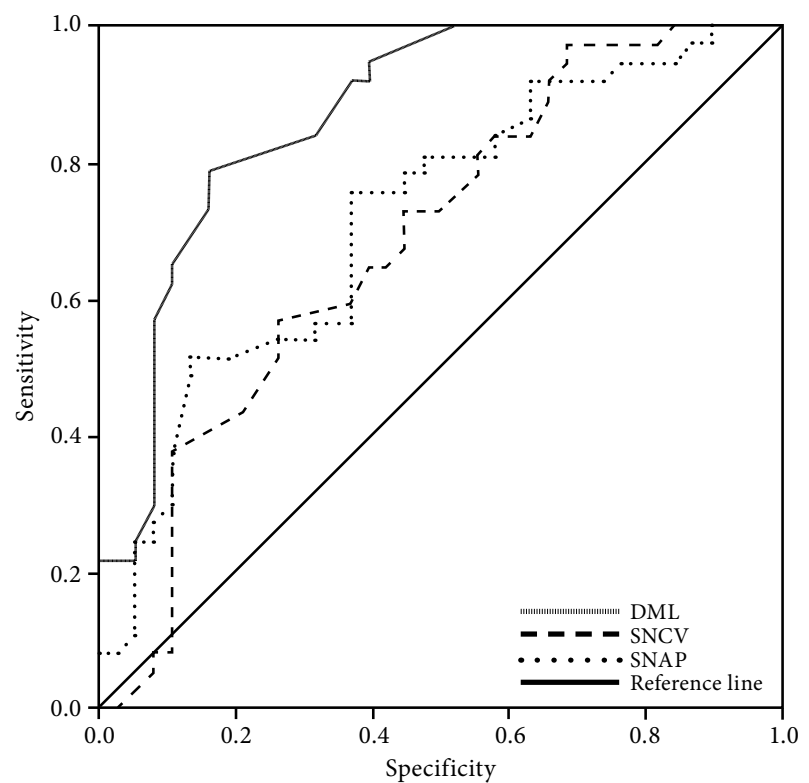

Figure 1. Receiver operating characteristic curves for DML, SNCV (palm-to-wrist) and, SNAP (palm-to-wrist) for distinguishing patients with and without spontaneous activity on EMG examination in CTS patients. Area under the curve (AUC) for DML, SNCV (palm-to-wrist) and, SNCV (palm-to-wrist) are 0.87 (95\% CI: 0.8-0.95), 0.68 (95\% CI: $0.56-0.81$ ) and 0.71 (95\% CI: 0.6-0.8); respectively.

DML: Distal motor latency; SNCV: Sensory nerve conduction velocity; SNAP: Sensory nerve action potential; EMG: Electromyography; CI: Confidence interval. significant independent risk variable in determining presence or absence of spontaneous activity on the needle EMG. If the median DML was $>4.9 \mathrm{~ms}$, the relative risk of detection of spontaneous activity on thenar muscle needle EMG was 13.5 (95\% CI: 3.6-51.2), even after controlling for other confounding factors. At the same time, for every $1 \mathrm{~ms}$ increase in the DML, the probability of having spontaneous activity on thenar muscle needle EMG increased by 7.2 times (95\% CI: 2.1-24.3).

\section{DISCUSSION}

In our study, we found that DML was the most determinative parameter for predicting the presence of spontaneous activity in patients with mild and moderate CTS with normal CMAP amplitude. No spontaneous activity was observed in any of the patients with normal DML. We found the most optimal cut-off value for median DML to be $4.9 \mathrm{~ms}$ in discriminating the presence of spontaneous activity on thenar muscle needle EMG. In the group of DML of $>4.9 \mathrm{~ms}$, the presence of spontaneous activity was 13.5 times more likely than the group of DML of $\leq 4.9 \mathrm{~ms}$.

Detecting the axonal degeneration in CTS patients is of utmost importance, as it can change the treatment 
options. ${ }^{[14,15]}$ The NCSs alone may be insufficient to detect the evidence of axonal loss in CTS patients, particularly with normal CMAP amplitude. The CMAP amplitude within normal limits does not does not always indicate the absence of the axonal degeneration. The presence of fibrillation potentials or neurogenic motor unit potentials (MUPs) in needle EMG are the most obvious evidence of axonal degeneration. ${ }^{[3,11,18]}$ There are many grading scales for the severity of CTS and these scales are mostly based on NCS variables. ${ }^{[19-21]}$ The benefit of thenar muscle needle EMG in electrophysiological evaluation of CTS is still controversial. Wee ${ }^{[22]}$ reported that needle EMG should not be performed routinely. Also, Mazur ${ }^{[17]}$ emphasized that thenar muscle EMG provided a minor additional contribution to NCSs in typical CTS patients. On the contrary, Werner and Albers ${ }^{[16]}$ found that the sensitivity of median motor and sensory NCSs for predicting an abnormality of needle thenar EMG was low (57 to 68\%) and that the information from thenar muscle EMG could not be predicted from the NCS.

In the current study, we found spontaneous activity in $31.4 \%$ of the patients at the thenar muscle. In the literature, the incidence of thenar muscle EMG abnormalities, including MUP changes, in CTS patients varies from 48 to $96 \%{ }^{[16,23-25]}$ However, the incidence of only denervation potentials in thenar muscles is 11 to $42 \% .^{[24-28]}$ The discrepancy in the incidence of thenar muscle needle EMG abnormalities may be related to the difference in the severity of CTS patients in the studies. Werner and Albers ${ }^{[16]}$ found EMG abnormalities (fibrillation and MUP changes) in $48 \%$ and spontaneous activity in $22 \%$ of the patients with CTS. In our study, we limited the needle EMG abnormalities to presence or absence of spontaneous activity, which is the most objective sign of axonal degeneration.

The mean age and symptom duration of the CTS patients with and without spontaneous activity were similar in our study. El-Emary and Hassan ${ }^{[26]}$ found that patients with spontaneous activity were significantly older and had a longer disease duration. Werner and Albers ${ }^{[16]}$ reported that there was a $2 \%$ increase risk for an abnormal needle EMG examination for each additional year of life. The authors evaluated acute and chronic axonal degeneration needle EMG abnormalities (spontaneous activity, increased MUP amplitude or polyphagia) together in their study. However, in the present study, we only evaluated acute axonal degeneration with spontaneous activity and this may have caused a discrepancy in the relationship between the needle EMG and both age and symptom duration.

Most studies investigating the electrophysiological findings in CTS have shown a correlation between the CMAP amplitude and thenar muscle needle EMG. ${ }^{[22,25-28]}$ Although we did not include patients with low CMAP amplitude in our study to determine predictors other than CMAP amplitude, we found that the CMAP amplitude was lower in patients with spontaneous activity. In a study including 229 CTS patients of whom $43 \%$ had low CMAP amplitudes, the CMAP amplitude was the most important indicator in detecting the presence of spontaneous activity. ${ }^{[27]}$ The authors reported that the rate of spontaneous activity was $>95 \%$, when the median CMAP amplitude was $\leq 2.1 \mathrm{mV}$. On the other hand, Werner and Alber ${ }^{[16]}$ found that low SNAP was more determinative than low CMAP in demonstrating axonal degeneration; however, in their study, CMAP amplitudes were within the normal limits for most cases. Therefore, determining the predictors of electrophysiological findings other than CMAP amplitude is valuable, particularly in patients with relatively normal CMAP amplitude.

In our study, spontaneous activity was not observed in any patients with normal DML. On the other hand, $49.3 \%$ of 75 CTS patients with prolonged DMLs had spontaneous activity. In a study from which patients with low CMAP amplitude were excluded, thenar muscles needle EMG abnormalities were found in $12 \%$ of patients with normal DML and in $32.6 \%$ of patients with prolonged DML. ${ }^{[18]}$ Although patients with low CMAP amplitude were not included in this study, the authors found that patients with needle EMG abnormalities had significantly lower CMAP amplitudes than patients without needle EMG abnormalities. In another study including 228 CTS patients, $52 \%$ of the patients showed needle EMG pathologies, including abnormalities in the recruitment pattern. ${ }^{[29]}$ Needle EMG abnormalities were seen in only $3.9 \%$ of patients with normal DML. On the other hand, there were EMG abnormalities in all patients with DML of $>7 \mathrm{~ms}$. In the aforementioned study, DMLs of $68 \%$ of the patients were prolonged and the severity of needle EMG findings was correlated with the degree of median motor DML values.

In the present study, we found significant differences in the DMLs, CMAP amplitudes, pwSNAP 
amplitudes, and pwSNCVs between CTS patients with and without spontaneous activity. Also, the absence or decreased amplitude of the 2dSNAP increased the odds ratio of presence of spontaneous activity. In addition, according to the ROC analysis, the DML, pwSNAP amplitude, and pwSNCV measurements were statistically significant in discriminating patients with and without spontaneous activity. We established the most optimal cut-off values in discriminating the presence of spontaneous activity for each parameter (DML $>4.9 \mathrm{~ms}$, pwSNAP amplitude $<22.5 \mu \mathrm{V}$ and pwSNCV $<25.6 \mathrm{~m} / \mathrm{s}$ ). On the other hand, the multiple logistic regression analysis revealed that DML was a significant independent risk variable in determining presence or absence of spontaneous activity on the needle EMG. We found that the patients of DML of $>4.9 \mathrm{~ms}$ were 13.5 times more likely to have spontaneous activity than those of DML of $\leq 4.9 \mathrm{~ms}$. At the same time, every $1 \mathrm{~ms}$ prolongation of DML increased the probability of spontaneous activity by seven times. In their study, Vennix et al. ${ }^{[28]}$ found that the sensitivity of DML to predict the presence of spontaneous activity was more than $95 \%$ while performing a needle examination of the APB in patients, if the median DML was $>4.5 \mathrm{~ms}$. Werner and Albers $^{[16]}$ observed a strong correlation between DML and axonal degeneration and an OR of 1.55 for the median motor latency indicated a 55\% increased risk for each increase of $1 \mathrm{~ms}$ in the motor latency. This ratio of the median/ulnar CMAP amplitude has not been evaluated much in the literature. Since we did not include severe cases with low CMAP amplitude in our study, we believe that the ratio of the median to the ulnar CMAP amplitude may be useful in the decision of needle EMG in our study population. We found that the ratio of the median/ulnar CMAP amplitude was lower in patients with spontaneous activity than patients without of spontaneous activity. On the other hand, when we performed subgroup analysis in patients with prolonged DML, we observed no significant relationship with the presence of spontaneous activity. Similarly, Werner and Albers ${ }^{[16]}$ found no significant relationship between the ratio of the median to the ulnar CMAP amplitude and needle EMG abnormalities.

Nonetheless, there are some limitations to this study. First, it has a small sample size which may limit the power of this study to generalize its findings. Second, we limited the needle EMG abnormality to the presence of spontaneous activity for determining the presence of axonal degeneration. In further studies, severity of spontaneous activity and MUP abnormalities may also be included in the assessment of axonal injury.

In conclusion, our study suggests that DML is the most important parameter for predicting the presence of spontaneous activity in patients with mild and moderate CTS with normal CMAP amplitude. In addition, the sensory parameters seem to be related in discriminating the presence of spontaneous activity. As we observed no spontaneous activity in any patients with normal DML in our study, we cannot recommend to perform needle EMG to thenar muscles in CTS patients with normal or even slightly prolonged DML. We believe that it would be beneficial to take into account DML primarily to detect axonal degeneration in earlier stages, considering that the probability of finding spontaneous activity is significantly increased in patients with a median DML of $>4.9 \mathrm{~ms}$.

\section{Declaration of conflicting interests}

The authors declared no conflicts of interest with respect to the authorship and/or publication of this article.

Funding

The authors received no financial support for the research and/or authorship of this article.

\section{REFERENCES}

1. Genova A, Dix O, Saefan A, Thakur M, Hassan A. Carpal tunnel syndrome: A Review of literature. Cureus 2020;12:e7333.

2. Wright AR, Atkinson RE. Carpal tunnel syndrome: An update for the primary care physician. Hawaii J Health Soc Welf 2019;78(11 Suppl 2):6-10.

3. Wang L. Electrodiagnosis of carpal tunnel syndrome. Phys Med Rehabil Clin N Am 2013;24:67-77.

4. Atroshi I, Englund M, Turkiewicz A, Tägil M, Petersson IF. Incidence of physician-diagnosed carpal tunnel syndrome in the general population. Arch Intern Med 2011;171:943-4.

5. Feng B, Chen K, Zhu X, Ip WY, Andersen LL, Page P, et al. Prevalence and risk factors of self-reported wrist and hand symptoms and clinically confirmed carpal tunnel syndrome among office workers in China: A cross-sectional study. BMC Public Health 2021;21:57.

6. Roh YH, Chung MS, Baek GH, Lee YH, Rhee SH, Gong HS. Incidence of clinically diagnosed and surgically treated carpal tunnel syndrome in Korea. J Hand Surg Am 2010;35:1410-7.

7. Mondelli M, Giannini F, Giacchi M. Carpal tunnel syndrome incidence in a general population. Neurology 2002;58:289-94.

8. Basiri K, Katirji B. Practical approach to electrodiagnosis of the carpal tunnel syndrome: A review. Adv Biomed Res 2015;4:50.

9. Sucher BM, Schreiber AL. Carpal tunnel syndrome diagnosis. Phys Med Rehabil Clin N Am 2014;25:229-47. 
10. Wipperman J, Goerl K. Carpal tunnel syndrome: Diagnosis and management. Am Fam Physician 2016;94:993-9.

11. Werner RA, Andary M. Electrodiagnostic evaluation of carpal tunnel syndrome. Muscle Nerve 2011;44:597-607.

12. American Association of Electrodiagnostic Medicine, American Academy of Neurology, and American Academy of Physical Medicine and Rehabilitation. Practice parameter for electrodiagnostic studies in carpal tunnel syndrome: Summary statement. Muscle Nerve 2002;25:918-22.

13. Wang L. Guiding treatment for carpal tunnel syndrome. Phys Med Rehabil Clin N Am 2018;29:751-60.

14. Keith MW, Masear V, Amadio PC, Andary M, Barth RW, Graham B, et al. Treatment of carpal tunnel syndrome. J Am Acad Orthop Surg 2009;17:397-405.

15. Atroshi I, Gummesson C, Johnsson R, McCabe SJ, Ornstein E. Severe carpal tunnel syndrome potentially needing surgical treatment in a general population. J Hand Surg Am 2003;28:639-44.

16. Werner RA, Albers JW. Relation between needle electromyography and nerve conduction studies in patients with carpal tunnel syndrome. Arch Phys Med Rehabil 1995;76:246-9.

17. Mazur A. Role of thenar electromyography in the evaluation of carpal tunnel syndrome. Phys Med Rehabil Clin N Am 1998;9:755-64.

18. Rubin DI, Dimberg EL. Needle EMG of thenar muscles in less severe carpal tunnel syndrome. J Clin Neurophysiol 2018;35:481-4.

19. Padua L, LoMonaco M, Gregori B, Valente EM, Padua R, Tonali P. Neurophysiological classification and sensitivity in 500 carpal tunnel syndrome hands. Acta Neurol Scand 1997;96:211-7.
20. Stevens JC. AAEM minimonograph \#26: The electrodiagnosis of carpal tunnel syndrome. American Association of Electrodiagnostic Medicine. Muscle Nerve 1997;20:1477-86.

21. Bland JD. A neurophysiological grading scale for carpal tunnel syndrome. Muscle Nerve 2000;23:1280-3.

22. Wee AS. Needle electromyography in carpal tunnel syndrome. Electromyogr Clin Neurophysiol 2002;42:253-6.

23. Marinacci AA. Comparative value of measurement of nerve conduction velocity and electromyography in the diagnosis of carpal tunnel syndrome. Arch Phys Med Rehabil 1964;45:548-54.

24. Stevens JC. AAEE minimonograph \#26: The electrodiagnosis of carpal tunnel syndrome. Muscle Nerve 1987;10:99-113.

25. Kimura I, Ayyar DR. The carpal tunnel syndrome: Electrophysiological aspects of 639 symptomatic extremities. Electromyogr Clin Neurophysiol 1985;25:151-64.

26. El-Emary WS, Hassan MM. Needle electromyography in carpal tunnel syndrome: Is it valuable or predictable? Egypt Rheumatol Rehabil 2016;43:41-6.

27. Chang CW, Lee WJ, Liao YC, Chang MH. Which nerve conduction parameters can predict spontaneous electromyographic activity in carpal tunnel syndrome? Clin Neurophysiol 2013;124:2264-8.

28. Vennix MJ, Hirsh DD, Chiou-Tan FY, Rossi CD. Predicting acute denervation in carpal tunnel syndrome. Arch Phys Med Rehabil 1998;79:306-12.

29. Murga L, Moreno JM, Menéndez C, Castilla JM. The carpal tunnel syndrome. Relationship between median distal motor latency and graded results of needle electromyography. Electromyogr Clin Neurophysiol $1994 ; 34: 377-83$ 